INTEGRACIÓN Y TIC EN HOTELES: IMPORTANCIA DE SU IMPLEMENTACIÓN

CONJUNTA

Revista Economía y Administración, Vol. 9, No. 1, 2018

\title{
INTEGRACIÓN Y TIC EN HOTELES: IMPORTANCIA DE SU IMPLEMENTACIÓN \\ CONJUNTA
}

\section{(1) Javier Del Cid Carrasco}

Universidad Tecnológica Centroamericana UNITEC

Posgrado/ Doctorado en Economía y Empresa Col La Campaña, Edificio CEUTEC, 5 to piso, oficinas académicas, Tegucigalpa,

ORCID: 0000-0002-1593-0491 javier.delcid@unitec.edu.hn

Cesar H. Ortega Jiménez

Universidad Nacional Autónoma de Honduras UNAH Facultad de Ciencias Economicas, Administrativas y Contables (FCEAC) Instituto de Investigaciones Economicas y Sociales (IIES) Ciudad Universitaria, edificio C2, primer piso, Tel/fax: 2216-6100 Ext. 100894

ORCID: 0000-0003-1911-9725

\section{DOI: $\underline{\text { http://dx.doi.org/10.5377/eya.v9i1.6652 }}$}

cortega@unah.edu.hn

\section{RESUMEN}

La presente es una investigación de enfoque cualitativo que utiliza la revisión sistemática de publicaciones científicas, con el fin de analizar las evidencias de diferentes industrias 0 sectores empresariales enfocados en aplicar estrategias de integración. Se delimita entre Integración Interna e Integración Externa y el uso de las Tecnologías de Información y Comunicación para dichos procesos.
Palabras Clave: Cadena de suministros, Tecnologías de información y comunicación (TIC), Integración, desempeño empresarial.

(1) Autor de contacto 
INTEGRACIÓN Y TIC EN HOTELES: IMPORTANCIA DE SU IMPLEMENTACIÓN CONJUNTA

Revista Economía y Administración, Vol. 9, No. 1, 2018

\title{
INTEGRATION AND ICT IN HOTELS: IMPORTANCE OF ITS JOINT IMPLEMENTATION
}

\begin{abstract}
(1) Javier Del Cid Carrasco Universidad Tecnológica Centroamericana UNITEC

Posgrado/ Doctorado en Economía y Empresa Col La Campaña, Edificio CEUTEC, 5to piso, oficinas académicas, Tegucigalpa, Honduras ORCID: 0000-0002-1593-0491

javier.delcid@unitec.edu.hn

Cesar H. Ortega Jiménez

Universidad Nacional Autónoma de Honduras UNAH Facultad de Ciencias Economicas, Administrativas y Contables (FCEAC) Instituto de Investigaciones Economicas y Sociales (IIES) Ciudad Universitaria, edificio C2, primer piso, Tel/fax: 2216-6100 Ext. 100894 ORCID: 0000-0003-1911-9725

DOI: DOI: http://dx.doi.org/10.5377/eya.v9i1.6652

cortega@unah.edu.hn
\end{abstract}

strategies. It is delimited between Internal Integration and External Integration and the use of Information and Communication Technologies for said processes.

Keywords: Supply chain, Information and communication technologies (ICT), Integration, business performance.

(1) Corresponding author 


\section{INTEGRACIÓN Y TIC EN HOTELES: IMPORTANCIA DE SU IMPLEMENTACIÓN \\ CONJUNTA}

Revista Economía y Administración, Vol. 9, No. 1, 2018

\section{INTRODUCCIÓN}

El eficaz desempeño de una empresa, del rubro que sea, estará ligado a diferentes condiciones del entorno, tanto del interno como del externo. Prácticas en la gestión de operaciones, tal como la aplicación y uso de Tecnologías de Información y comunicación, en las empresas han sido estudiadas desde diferentes puntos de vista.

Para efectos de esta investigación las tomaremos como herramientas que apoyan a cualquier estrategia que la empresa haya planteado, estrategias como las de Integración de la cadena de suministros necesitan de mucha tecnología para poder realizarse de manera eficiente, al menos es lo que lógicamente pensamos.

Justo ese enfoque es la tarea principal de esta investigación, encontrar evidencias previas, de estudios que se han realizado ya sea en el sector de manufactura y bienes, así como en empresas de servicio acerca de estos dos términos y su complementariedad para el alcance de objetivos. Lo que nos permita responder la siguiente pregunta:

¿Existe evidencia empírica que demuestre qué la empresa puede obtener mejor desempeño a través de la integración de la cadena de suministros? ¿Los procesos de integración deben ser implementados con el uso de Tecnologías de Información y Comunicación (TIC)?

En la actualidad, se acepta ampliamente que la economía mundial ha flotado y es cada vez más compatible con las TIC que nunca (Rodriguez-Rodriguez \& Leon, 2016). Las fuerzas de globalización han cambiado nuestra forma de hacer negocios. Las empresas se enfocan cada vez más en sus capacidades básicas y la tendencia hacia la externalización ha crecido (por ejemplo, Clemons et al., 1993, Doh, 2005, Espino-Rodríguez y Padron-Robaina, 2006, Mol et al. Muchas firmas han comenzado a subcontratar sus actividades principales de la cadena de valor que van desde no sólo las funciones de fabricación sino también a sus desarrollos de productos (Jean, 2007).

Por medio de un cuadro comparativo evaluaremos diferentes artículos de revistas especializadas en Gestión de operaciones, Gestión Logística, Administración de hoteles y cualquier tema que abarque estas prácticas. De dichos artículos nos interesa identificar cuáles son las variables, proposiciones, 


\section{INTEGRACIÓN Y TIC EN HOTELES: IMPORTANCIA DE SU IMPLEMENTACIÓN \\ CONJUNTA}

Revista Economía y Administración, Vol. 9, No. 1, 2018

diferentes países del mundo, no sólo del

preguntas de investigación hechas en torno a nuestros tópicos, así como, hallazgos y conclusiones de dichos estudios.

Este documento se dividirá en Marco de Referencia y Proposiciones, Metodología, Discusión de Resultados y Conclusiones.

\section{MARCO DE REFERENCIA Y PROPOSICIONES}

La necesidad de esta investigación nace en el marco del proyecto PAR - Bienes y Servicios proyecto en el cual se estará dando seguimiento y mayor profundidad a temas altamente discutidos y estudiados desde hace algunas décadas, enfocados en la competitividad, estrategias organizacionales, nuevas prácticas de la gestión de operaciones; áreas en donde aún hay muchas ventanas de análisis y discusión, tal como lo afirman en la memoria técnica del proyecto denominado “COMPETITIVIDAD Y PRODUCTIVIDAD: PRODUCCIÓN (BIENES Y SERVICIO) DE ALTO RENDIMIENTO (PAR)" (Del Cid Carrasco, 2017): "Esta investigación permanente para obtener cada vez más altos rendimientos de producción, continúa y promete seguir atrayendo multitud de directivos y académicos de área de gestión de dirección de producción/operaciones, sino también de toda la comunidad de administración de empresas y de ingeniería en general." (Ortega Jimenez, 2016).

Tres niveles distintos y sus interrelaciones describen los conceptos que se proponen para la definición de PAR: nivel de procesos (producción de bienes y servicios), nivel de productos y servicios, así como nivel de la unidad de negocios. Dichos niveles pueden observarse en el marco referencial presentado en la Figura 2.1 .

Figura 2.1 Marco de Referencia Proyecto PAR (Bienes y Servicios)

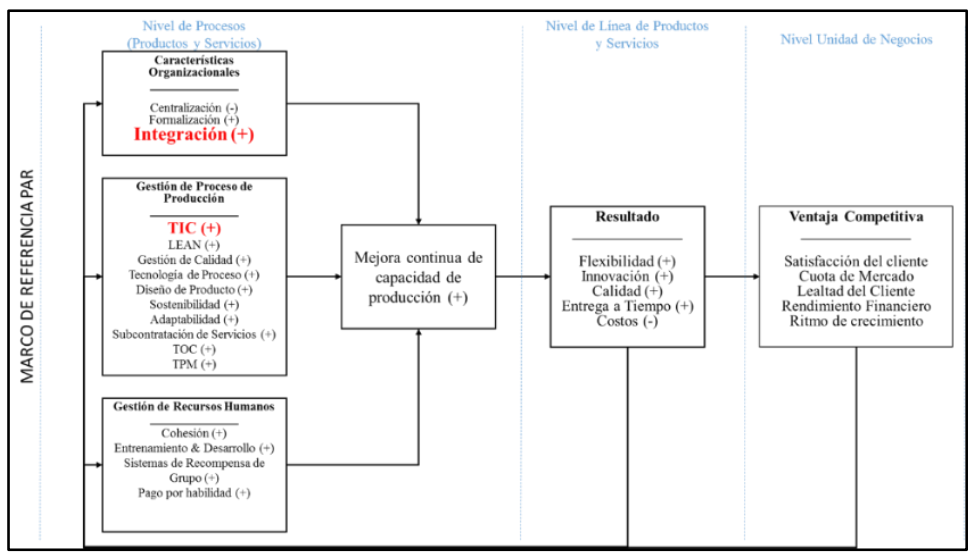

Fuente: Instituto de Investigaciones Económicas y Sociales.

En efecto el estudio se centrará en dos de dichas variables, TIC e Integración. De estas variables encontramos mucha 


\section{INTEGRACIÓN Y TIC EN HOTELES: IMPORTANCIA DE SU IMPLEMENTACIÓN \\ CONJUNTA}

Revista Economía y Administración, Vol. 9, No. 1, 2018

literatura, de la cual se pretende encontrar

evidencia empírica acerca del desempeño de la implementación dentro de una empresa. No vista desde la perspectiva de los tipos de estrategia, niveles de implementación y otras, sino, desde su aporte comprobado para el alcance de sus objetivos.

En la búsqueda de teoría e investigaciones que permitieran determinar el estado de arte y la evidencia de la información que esperamos construir, encontramos algunas publicaciones como la siguiente que hace referencia a los procesos de integración:

La gestión de la calidad ha recibido una atención creciente en la gestión de la cadena de suministro. El concepto de la integración de la calidad de la cadena de suministro (SCQI) se utiliza para explicar cómo un fabricante focal se integra con sus proveedores de aguas arriba y clientes de aguas abajo para la mejora de la calidad (Huo, Ye, \& Zhu, 2016)

Aunque este estudio contribuye a la literatura y la práctica, varias limitaciones ofrecen oportunidades para futuros estudios. Primero, como hemos discutido, la evidencia empírica relativa al SCQM es inconsistente. Estudios futuros pueden explorar esta área introduciendo más mediadores y moderadores. (Huo, Ye, \& Zhu, 2016)

Así mismo encontramos estudios acerca de TIC y sus implementaciones en los procesos de integración y aporte a los resultados esperados. A continuación, una de las conclusiones de un estudio enfocado en las TIC y su impacto en el valor de marca y lealtad del cliente:

Sin embargo, debido a que las dudas sobre la eficiencia de las soluciones tecnológicas parecen persistir, son necesarias nuevas evidencias sobre la capacidad de las TIC. Además, el valor de la marca de hotel ha comenzado a ser investigado empíricamente sólo en los últimos años, y principalmente en los países asiáticos, lo que revela que el concepto necesita nuevas perspectivas en la nueva configuración del hotel. Por último, si bien los temas de las TIC y de la equidad de la marca recibieron un interés considerable en la industria de la hospitalidad cuando se estudian por separado, existe una brecha en cuanto al impacto que las TIC podrían ejercer

\section{sobre el valor de la marca hotelera} basada en el cliente. El objetivo del presente estudio fue llenar las brechas antes mencionadas proporcionando una investigación empírica sobre ambos 


\section{INTEGRACIÓN Y TIC EN HOTELES: IMPORTANCIA DE SU IMPLEMENTACIÓN \\ CONJUNTA}

Revista Economía y Administración, Vol. 9, No. 1, 2018

constructos (Šeric, Gil-Saura, \& Alejandro, 2016).

Un artículo enfocado en determinar el estado del arte en el tópico de los CRM siglas de Administración de Relaciones con Cliente (Customer Relationship Management), concepto que alineamos como parte de las herramientas para una integración con el cliente. Los autores en sus conclusiones y vacíos de la información proponen:

Además, invitamos a los investigadores a investigar los patrones de los sistemas de CRM y sus usos en las industrias y los países para ver cómo las características del entorno empresarial (cultura nacional, el nivel de competitividad, la turbulencia de la industria, etc.) para los procesos de creación de conocimiento del cliente. Dada la importancia del intercambio de datos como un vínculo entre el aprendizaje organizacional y la calidad de los datos, la calidad de los datos media la relación entre el intercambio y el desempeño, por lo que se necesita trabajo futuro para ampliar el dominio del intercambio de datos (Soltani \& Navimipour, 2016).

Con dichos antecedentes, al igual que los hallazgos que hagamos al finalizar esta investigación, nos enfocaremos en el vacío de información existente en la siguiente temática: El impacto de una estrategia de integración con implementación de TIC para los resultados de una empresa. Enfocado en el sector hotelero.

\section{METODOLOGÍA}

Una revisión literaria inicial se considera la parte exploratoria del diseño de esta investigación. Se establecieron criterios sistemáticos de búsqueda por medio de palabras claves o términos claves como: ICT, TIC, SCM, Integración, Hoteles, entre otros.

Se seleccionaron cerca de 123 artículos. Los que luego pasaron por un proceso de evaluación y selección final. Este filtro nos llevó a seleccionar 20 artículos que reunieran o demostrasen los procesos más robustos y con enfoques alineados cercanamente con los criterios buscados. Se utilizaron solamente artículos publicados en revistas indexadas con procesos de revisión literarios comprobados. Se propone construir una tabla comparativa conteniendo la siguiente información: Título del artículo, Referencia bibliográfica, Tipo de artículo, Población, Muestra, Fuentes de información, Enfoque Teórico, 


\section{INTEGRACIÓN Y TIC EN HOTELES: IMPORTANCIA DE SU IMPLEMENTACIÓN CONJUNTA}

Revista Economía y Administración, Vol. 9, No. 1, 2018
Proposiciones o preguntas de
investigación, Variables, Resultados y
Conclusiones.
- Implementación y existencia de TIC
- Niveles de implementación de las TIC

De estos artículos discutiremos acerca de su forma de diagramar las relaciones entre variables, sus resultados y aportes para nuestras conclusiones.

\section{DISCUSIÓN \\ RESULTADOS}

DE

Siguiendo la metodología propuesta en el epígrafe anterior los análisis presentados a continuación son respaldados a través de información contenida en la tabla comparativa para revisión sistemática de literatura que podrán consultar en los cuadros anexos de este documento (Ver Anexo A, Tabla A1). Por lo que encontraremos en este segmento serán resúmenes o discusiones de como los diferentes artículos discutieron, enfocaron y analizaron las diferentes variables de interés para esta investigación:

- Integración: Interna, con Clientes y con proveedores.

- Niveles de Integración: de Información, operativa y financiera

- Desempeño de la compañía

\subsection{Integración}

De los artículos evaluados todos realizan un enfoque de proposiciones o preguntas de investigación enfocadas en cuál es el rol de las estrategias y prácticas de integración en cualquier nivel y dirección de esta como variables que influyen en el desempeño de la empresa. Por ejemplo, lo planteado por los siguientes autores, "La feroz competencia entre hoteles e incluso destinos enteros está precipitando la necesidad de innovación en tecnología, organización y estrategia. En un esfuerzo por estimular la innovación entre los hoteleros, este estudio consideró temas que merecen más atención en la investigación de hospitalidad, es decir, TQM habilitado para TI, CRM y SCM, especialmente en un lugar poco estudiado y de rápido crecimiento como los Emiratos Árabes Unidos." (Daghfous \& Barkhi, 2009); en la afirmación anterior ya se genera una primera referencia al SCM siglas para la Administración de la cadena de suministros por su nombre en inglés Supply Chain Management y a las TIC como es el caso del CRM siglas para el 


\section{INTEGRACIÓN Y TIC EN HOTELES: IMPORTANCIA DE SU IMPLEMENTACIÓN \\ CONJUNTA}

Revista Economía y Administración, Vol. 9, No. 1, 2018

\section{Customer Relationship Management o la}

Administración de relaciones con el

cliente. Esto nos da un primer indicio de integración hacia el cliente apoyado también de sistemas de información y de integración con proveedores e interna por medio de la cadena de suministros.

\subsubsection{Integración interna}

Dentro de las variantes o tipos de integración la primera categoría propuesta que analizaremos es la Integración Interna de la empresa haciendo énfasis en las interacciones entre áreas.

Como gerentes de empresas surgen preguntas acerca de cómo integrar de forma eficiente los equipos de trabajo. ¿Cómo se podrían formar equipos de alto rendimiento? ¿Cuáles son los factores que tomar en cuenta?

Algunos investigadores se han enfocado en uno de los factores que sin duda no puede faltar en un proceso de investigación, la comunicación. Enfocados en la comunicación como competencia los autores de este artículo de enfoque cualitativo afirman: "Por tanto, al identificar de forma concreta a la comunicación como un proceso, compete señalar que este de igual forma representa el intento de unificar dos organismos, por medio de la emisión y recepción de mensajes que tengan un significado para ambos (Argueta \& Martel, 2014).”

Sin embargo, para evaluar el proceso de la integración debemos evaluar también factores como el tamaño de la empresa, objetivos de dicho proceso, percepción de los empleados, entre otros.

El siguiente artículo se enfoca en los departamentos de logística y de marketing de las empresas de manufactura afirmando que: "Se demostró que las interacciones frecuentes y la integración entre los departamentos de marketing y logística en una empresa están estrechamente relacionada con la percepción de los gerentes de que existe una mejor sinergia y eficacia en las relaciones interdepartamentales conduciéndoles a un mejor desempeño del área. (Stank, Daugherty, \& Ellinger, 1999)"

Documento que basó sus escalas principales para esta conclusión en indicadores para medir la cantidad de interacciones que se generaban entre cada departamento. Demostrando así su principal conclusión de que "Empresas con mayores niveles de integración interna demuestran mejor desempeño que aquellas 


\section{INTEGRACIÓN Y TIC EN HOTELES: IMPORTANCIA DE SU IMPLEMENTACIÓN \\ CONJUNTA}

Revista Economía y Administración, Vol. 9, No. 1, 2018

con menores niveles de integración

interna. (Stank, Daugherty, \& Ellinger, 1999)".

Otro enfoque que le podemos brindar a la Integración Interna de la empresa es aquella provocada por las fusiones de las empresas donde se lleva a cabo un proceso de integración de nuevos equipos de trabajo y debe ejecutarse de forma rápida y con las herramientas necesarias.

La industria hotelera es una de las de mayor crecimiento y más fuerte dentro del rubro de turismo. Este estudio que se realizó con empleados de 15 firmas de hoteles globales en Reino Unido enfocado en las reacciones o sentimientos de los empleados de una empresa (Hotel) acerca de la fusión de dichas empresas:

"En términos generales, la consideración de las tres emociones más fuertemente sentidas de los empleados indicó que los encuestados tenían más probabilidades de enfocarse en aspectos desfavorables que los aspectos favorables o mixtos al explicar su elección de emociones. En general, el $81 \%$ de estas explicaciones se centraron en los aspectos negativos, ya que no hubo diferencias significativas entre los empleados adquiridos y los adquirentes (Saunders, Altinay, \& Riordan, 2009)”.
Este hallazgo demuestra como la fusión nos llevara a procesos difíciles de integración interna en la compañía lo que nos lleva a pensar en que se debe contar con todas las herramientas necesarias para generar el flujo esperado de dicho proceso con la vista puesta en la consecución de los objetivos de la empresa.

Sin duda, todos los estudios analizados han brindado datos demostrando la influencia de la integración interna sobre el alcance de objetivos de una empresa. Otro estudio realizado en empresas de manufactura plantea una proposición donde pretende demostrar que "H1a. La integración interna está positivamente relacionada con el rendimiento del fabricante dentro de una cadena de suministro." Y llegando a la siguiente conclusión Aceptada dicha proposición (Flynn, Huo, \& Zhao, 2010).

\subsubsection{Integración con proveedores}

La integración cadena arriba como también podemos llamar a la integración con los proveedores, es otro factor de interés en este estudio. Diferentes investigaciones han presentado hallazgos al respecto como el siguiente estudio realizado en 116 plantas automotrices en Tailandia: 


\section{INTEGRACIÓN Y TIC EN HOTELES: IMPORTANCIA DE SU IMPLEMENTACIÓN \\ CONJUNTA}

Revista Economía y Administración, Vol. 9, No. 1, 2018

Este trabajo demuestra los beneficios de la conceptualización de la Integración de Cadena de suministro y el desempeño operacional como construcciones multidimensionales (Dröge et al., 2004, Koufteros et al, 2005, Devaraj et al, 2007, Swink et al., 2007, Flynn et al., 2010). A diferencia de algunos estudios previos que conceptualizan el Integración de Cadena de suministro y / o el desempeño operacional como construcciones unidimensionales (por ejemplo, Stank et al., 1999, Rosenzweig et al., 2003), este trabajo nos permite ver de manera comprensiva los detalles de las relaciones Integración de Cadena de suministro (Wong, Boon-Itt, \& Wong, 2011).

La interesante pregunta en estos casos es, ¿Quién debe dar inicio a un proceso de integración? Sin duda, es un proceso que fluirá naturalmente en cuanto al miembro más fuerte de la cadena de suministro lo exija.

\subsubsection{Integración con el cliente}

Si evaluamos desde el punto de vista de la razón de ser de una empresa, diríamos que esta es la integración de mayor importancia para toda empresa, pero definitivamente es solamente una pieza importante dentro de todo un proceso integrado.

"A pesar de que todos los hoteles declararon un enfoque centrado en el cliente para la implementación de CRM, los hoteles pequeños y grandes difieren significativamente en algunos motivos específicos, lo que indica claramente los diferentes problemas operativos y la situación gerencial de los hoteles. Analíticamente, todos los encuestados afirmaron que la mejora de la calidad del servicio y, por lo tanto, la satisfacción del cliente y la lealtad son las razones más importantes para seguir CRM, mientras que reportaron menos importancia en la implementación de CRM para aumentar la satisfacción del empleado y / o reducir los costos (Sigala, 2005)"

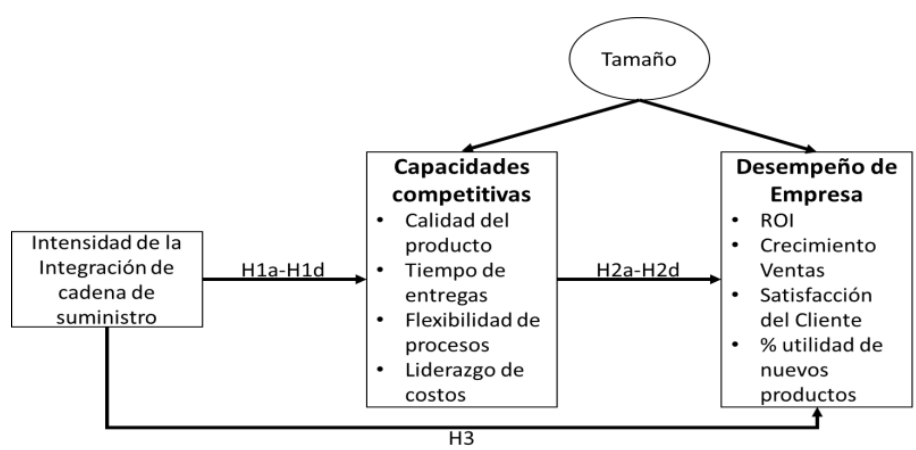

Vemos como empiezan a surgir términos donde implican la implementación de TIC dentro de procesos de integración. Sin embargo, como mencionan los autores, el costo de estos procesos son altos y muchas 


\section{INTEGRACIÓN Y TIC EN HOTELES: IMPORTANCIA DE SU IMPLEMENTACIÓN \\ CONJUNTA}

Revista Economía y Administración, Vol. 9, No. 1, 2018

veces se necesita de inversión por parte de

la empresa, pero al mismo tiempo por parte de los clientes. Es donde, si el cliente no percibe un beneficio de dicha integración, no fructificará dicho proceso.

\subsubsection{Intensidad y niveles de integración}

La intensidad y niveles de integración son otro factor que se debe revisar cuando estamos analizando dicha variable. Estudio realizado en empresas de producción de consumo masivo con empresas en más de 35 países por medio de entrevistas a gerentes de estas plantea un análisis con el siguiente marco referencial de acuerdo con sus hipótesis:

Figura No. 1 Modelo hipotético de la influencia de la intensidad de la

(Rosenzweig, Roth, \& Dean Jr., 2003)

Integración de la cadena de suministro en las capacidades competitivas y el desempeño empresarial

Este estudio establece teórica y empíricamente las formas en que la integración de la cadena de suministro conduce a aumentos en las capacidades competitivas y al mejoramiento del desempeño empresarial, proporcionando pruebas que anteriormente carecían de literatura.

Los resultados muestran que los fabricantes de productos de consumo con alta intensidad de integración logran una calidad de producto superior, confiabilidad de entrega, flexibilidad de proceso y liderazgo en costos.

Por lo tanto, la alta intensidad de la integración no sólo aumenta las capacidades, sino que también reduce los costos netos de la actividad empresarial mediante la interconexión de los elementos de la cadena de suministro, proporcionando esencialmente a los fabricantes un mayor apalancamiento en el mercado cada vez más volátil. (Rosenzweig, Roth, \& Dean Jr., 2003)

La subcontratación es otra estrategia por medio de la cual se cumple la integración de suministros. Se realizó un estudio enfocado a empresas que brindan servicios de procesos en subcontratación BPO con una muestra de 205 gerentes de estas empresas demostraron los siguientes comportamientos: 


\section{INTEGRACIÓN Y TIC EN HOTELES: IMPORTANCIA DE SU IMPLEMENTACIÓN \\ CONJUNTA}

Revista Economía y Administración, Vol. 9, No. 1, 2018

Figura No. 2. Modelo hipotético de (Narayanan, Jayaraman, Luo, \&

estudio de empresas BPO.

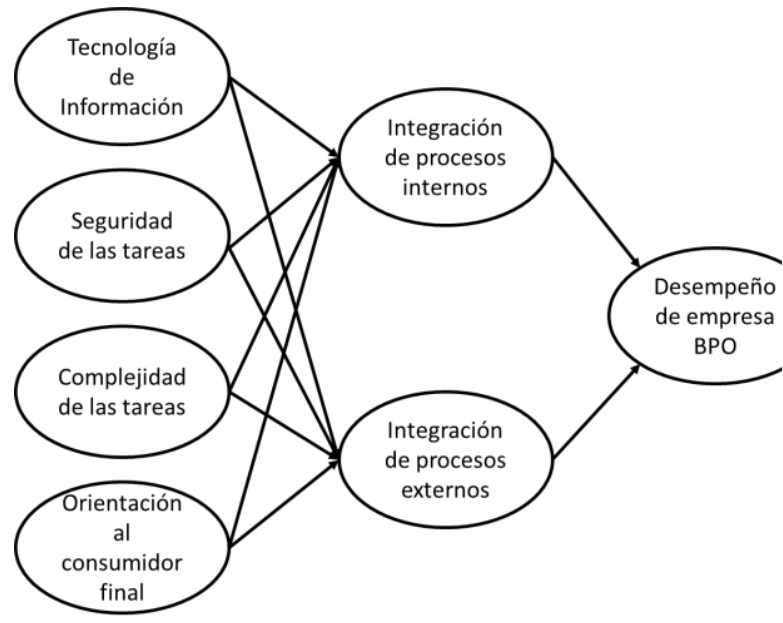

(Narayanan, Jayaraman, Luo, \& Swaminathan, 2011)

1. Presentamos primero modelo de subcontrataciones que identifica los antecedentes clave para la integración de procesos internos $\mathrm{y}$ externos $\mathrm{y}$ las consecuencias de dicha integración en el rendimiento de la empresa $\mathrm{BPO}$.

2. Finalmente, también se pueden obtener importantes ideas sobre nuestras variables de control. Nuestros resultados que la subcontratación inversa aumenta el rendimiento de BPO sugiere que las empresas de BPO que se centran en la capacidad de respuesta - mediante la localización de centros de entrega más cerca del cliente, tienen un rendimiento mejor que las empresas BPO que no.
Swaminathan, 2011)

\section{Tecnologías de información y comunicación (TIC)}

La segunda variable de relevancia a estudiar en esta investigación son las TIC. Este tema no solamente aplica a las prácticas empresariales. Conociendo que se refieren al uso de nuevas herramientas de comunicación y tecnologías, una afirmación de un artículo enfocado más en la educación e influencia social parece interesante:

De esto depende la productividad de las organizaciones-empresas las cuales basarán tal productividad en la innovación constante y permanente utilizando cada vez tecnologías más potentes. Por lo tanto, se vuelve necesario desde los diversos agentes económicos, entre ellos el sector académico-educativo preparar a que el educando logre irse adaptando e insertando apropiadamente para ser parte de la revolución digital (Raudales García, 2013).

Sin el afán de entrar en el campo de los profesionales que programan, crean o 


\section{INTEGRACIÓN Y TIC EN HOTELES: IMPORTANCIA DE SU IMPLEMENTACIÓN \\ CONJUNTA}

Revista Economía y Administración, Vol. 9, No. 1, 2018

diseñan estos sistemas, es importante también tener una perspectiva de esos. Donde se utilizan nuevos términos como herramientas para la inteligencia de los negocios, entre muchos.

"Todas las decisiones que se ejecutan en una empresa deberían provenir de las propias operaciones empresariales y de fenómenos que se producen en el entorno organizacional. Expresado todo en términos de información" (Araníbar S., 2003) por ello es necesario contar con un método de calidad para la gestión de las fuentes y tratamiento de su transformación (pasar de datos a información y luego conocimiento) hasta que se convierta en el insumo que alimente los sistemas de inteligencia de negocios. (Aguilar Romero \& Rodríguez García, 2016)”

Enfocándonos en el sector de hoteles y otras empresas de servicio encontramos algunas afirmaciones como: "Hoy en día, sería virtualmente imposible intentar funcionar un negocio de la hospitalidad sin ninguna tecnología. Sin importar como se usen las TIC, el no usarlas puede llevar una empresa de la hospitalidad a la quiebra. En la organización moderna de la hospitalidad, las TIC desempeñan un papel importante e integral en todo su entorno
(Connolly, Bustos R., \& Moreno L., 2008)".

La sistematización de los canales y formatos de comunicación en las empresas contemporáneas, agilizan el flujo de la información a lo largo de la misma, con la particularidad de la despersonalización de las relaciones interpersonales entre los asociados (Argueta \& Martel, 2014).

El enfoque de las TIC no nos permite encasillarlas como herramientas para una tarea en específico, dado que pueden ser adaptadas o creadas para simplificar y apoyar en la gestión de cada uno de los departamentos de cualquier empresa. Sin embargo, son herramientas de gran apoyo para que un proceso de integración se lleve a cabo.

Un estudio realizado en Croacia en la localidad de Dalmacia con todas las cadenas hoteleras de la zona enfocado a analizar la percepción de los clientes referente al uso de tecnología en dichos hoteles demostró una relación positiva entre el uso de las TIC y las comunicaciones integradas de marketing, IMC por su nombre en inglés Integrated Marketing Communications.

"La comprensión de la relación entre las TIC y el IMC debería alentar a los gerentes 


\section{INTEGRACIÓN Y TIC EN HOTELES: IMPORTANCIA DE SU IMPLEMENTACIÓN \\ CONJUNTA}

Revista Economía y Administración, Vol. 9, No. 1, 2018

a utilizar tecnologías avanzadas de manera

más efectiva en sus estrategias de comunicación. Las nuevas soluciones tecnológicas tienen un profundo impacto en la integración de las herramientas de comunicación, por lo que los gestores deben adoptar nuevas y mejores formas de entender, alcanzar y conectarse con los consumidores, que se encuentran en un mercado extremadamente fragmentado. Las inversiones en tecnologías avanzadas y de última generación y la consideración de la opinión de los huéspedes para su mejoramiento les ayudarán en la implementación de los programas IMC exitosos (Šerić \& Gil-Saura, 2012)”.

Lo anterior es un ejemplo claro de una tarea específica de la empresa apoyada con TICs. La mayor parte de estos estudios han demostrado que si existe una relación positiva entre la adaptación de las TIC y el rendimiento, sin embargo, encontramos un artículo que se refiere a esta relación como una ambigüedad sobre todo cuando incluyen una variable como el poder o tamaño de los proveedores.

"Relaciones entre el poder, los compradores y los proveedores - el impacto de las TIC para los pequeños proveedores: En los últimos años, ha habido una cantidad creciente de investigación sobre el impacto de las TIC en las relaciones y el desempeño de las empresas. Este tema se ha planteado en la comercialización (por ejemplo, Kim y otros, 2006), la cadena de suministro (por ejemplo, Sanders, 2005), los sistemas de información (por ejemplo, Rai et al., 2006) 2006).

Sin embargo, todavía hay pruebas fragmentadas sobre cómo las TIC reestructuran las relaciones entre empresas, especialmente en relación interorganizacional asimétrica, como entre las empresas multinacionales y sus proveedores. De hecho, no está claro si las TIC favorecen a los pequeños proveedores en su relación con las multinacionales o las ponen en desventaja (Jean, 2007)”.

Hay un modelo propuesto por los investigadores que lideraron un estudio en el cual dividieron la implementación de las Tecnologías de la información y comunicación en áreas funcionales de un hotel:

- División de Habitaciones

- Comidas y Bebidas

- Oficinas administrativas en general 


\section{INTEGRACIÓN Y TIC EN HOTELES: IMPORTANCIA DE SU IMPLEMENTACIÓN \\ CONJUNTA}

Revista Economía y Administración, Vol. 9, No. 1, 2018

- Tecnologías en la habitación.

De acuerdo con esa división se propusieron los siguientes criterios de evaluación de la intensidad o grado de implementación de las TIC de acuerdo con disponibilidad de los componentes, integración de los componentes y la intensidad de uso de las TIC. (Sirirak, Islam, \& $\mathrm{Ba}, 2011)$

La productividad operativa y la satisfacción del cliente son los indicadores de desempeño importantes en la industria hotelera (Johnston y Jones, 2004). La productividad se define como la relación de la producción real a la entrada durante un período de tiempo (Martin y Horne, 2001; Gronroos y Ojasalo, 2004; Arthur y Stefan, 2006). Por lo tanto, la productividad operativa se define como una función de la relación de los productos operativos a los insumos durante un período de tiempo en que los insumos incluyen los materiales, los equipos y los costos de personal y los productos incluyen ingresos, número de bienes o servicios vendidos y número de clientes procesados Johnston y Jones, 2004). (Sirirak, Islam, \& Ba, 2011)

Un término no incluido en el capítulo de integración fue el de Integración
Horizontal que se tomará en cuenta en otros estudios, pero en este caso fue utilizado por autores que realizaron una investigación a través de hoteles y cualquier organización de turismo en China donde pudieron concluir:"La industria china del turismo ha seguido una pauta similar en el desarrollo de las aplicaciones de las TIC como Europa y América. El desarrollo se inició en las líneas aéreas, y luego se extendió a los hoteles, operadores turísticos, atracciones y DMO, con los dos primeros sectores a la cabeza. Es cierto que las aerolíneas y los hoteles son relativamente maduros y en los últimos años se han desarrollado en términos de su adopción de TIC en la organización y la conciencia de las TIC.

Aunque se han producido algunos cambios en los operadores turísticos, las atracciones turísticas y las OGD, las aplicaciones de las TIC en estos sectores son limitadas. Los hallazgos de la investigación en este estudio exploran un panorama general del estado chino de desarrollo del turismo electrónico (Xiaoqiu Ma, Buhalis, \& Song, 2003)"

Un estudio realizado para la industria manufacturera automotriz plantea: "Esta investigación se centra en las siguientes 


\section{INTEGRACIÓN Y TIC EN HOTELES: IMPORTANCIA DE SU IMPLEMENTACIÓN \\ CONJUNTA}

Revista Economía y Administración, Vol. 9, No. 1, 2018

dos dimensiones: (a) implementación

efectiva del proceso, que evalúa si la fábrica implementa apropiadamente una nueva tecnología de fabricación después de obtenerla y (b) trabajando con proveedores de tecnología (implicación del proveedor), que sirve para evaluar si la fábrica está trabajando estrechamente con los proveedores para desarrollar tecnologías nuevas y apropiadas. Esta última dimensión abarca no sólo la tecnología de procesos, sino también la tecnología de los productos (Machuca, Ortega Jiménez, Pedro, \& Pérez Diéz de los Rios, 2011)

Este planteamiento anterior nos lleva a pensar en la necesidad que existe de un futuro estudio que trataremos en el siguiente capítulo.

\section{CONCLUSIONES}

Implicaciones teóricas

De acuerdo con lo discutido o absorbido de la literatura existente tenemos suficientes evidencias para saber que la práctica de integración es una necesidad para que las empresas obtengan un mejor rendimiento y eficacia de desempeño. Teóricamente podemos definir diferentes clasificaciones y niveles de integración dentro y en el entorno de la empresa. La integración no solamente se puede calificar entre integración de cadena de suministros sino también a lo interno de la compañía como interdepartamental y de equipos de colaboración.

Las TIC son de suma importancia para agilizar, apoyar y ejecutar de mejor forma cualquier operación dentro de las empresas. No tenemos un estudio empírico que demuestre el éxito o fracaso de una ejecución de procesos de integración de acuerdo con el uso o no de TICS.

\section{Implicaciones prácticas}

Realizar un enfoque del proceso de integración para hoteles, determinado por los departamentos involucrados e incluir el tema de integración horizontal como algunos autores lo hicieron para referirse a empresas del mismo nivel en la cadena de suministros que requieren generar integración.

Implícitamente, en todo proceso de implementación de una integración, ya sea vertical u horizontal, serán utilizadas herramientas tecnológicas y de comunicación. Las TIC por su lado, no son parte solamente de un proceso de integración, sino, son herramientas fundamentales 


\section{INTEGRACIÓN Y TIC EN HOTELES: IMPORTANCIA DE SU IMPLEMENTACIÓN \\ CONJUNTA}

Revista Economía y Administración, Vol. 9, No. 1, 2018

\section{Limitaciones como oportunidades de \\ BIBLIOGRAFÍA investigación futura}

El enfoque cualitativo de esta investigación y la falta de acceso momentáneamente a información cuantitativa y empírica de la industria hotelera en nuestro entorno no nos ha permitido realizar una evaluación de factores que nos permitan tener resultados concluyentes para contestar las siguientes preguntas de investigación:

- ¿Cuáles son los factores más influyentes en el desempeño de un proceso de integración? Costos, tiempo, complejidad de implementación, entre otros.

- ¿La implementación de un proceso de integración es más eficiente para los resultados de una empresa cuando se utilizan TIC o no?

\section{RECONOCIMIENTOS}

Esta investigación ha sido parcialmente financiada por el Proyecto PAR (IIES-UNAH). Los autores desean reconocer a UNAH por su apoyo.
Aguilar Romero, M., \& Rodríguez García, J. L. (2016). Comparación de opciones para Inteligencia de negocios en los principales sistemas gestores de bases de datos del mercado. Economía y Administración (E\&A), 5-20.

Argueta, J. D., \& Martel, J. R. (2014). ¿Es la comunicación en la empresa una competencia que debe desarrollarse? Revista Economía y Administración (E\&A), 26-64.

Connolly, D., Bustos R., L. E., \& Moreno L., R. (2008). LAS TIC (Tecnologías de la Información y las Comunicaciones) COMO ESTRATEGIA PARA LA INDUSTRIA DE LA HOSPITALIDAD. Scientia et Technica, 229-234.

Daghfous, A., \& Barkhi, R. (2009). The strategic management of information technology in UAE hotels: An exploratory study of TQM, SCM and CRM implementations. Technovation, 588-595. 


\section{INTEGRACIÓN Y TIC EN HOTELES: IMPORTANCIA DE SU IMPLEMENTACIÓN CONJUNTA}

Revista Economía y Administración, Vol. 9, No. 1, 2018

Del Cid Carrasco, J. E. (2017). Examen de candidatura . Tegucigalpa.

Fabbe-Coste, N., \& Jahre, M. (2008). Supply chain integration and performance: a review of the evidence. The international Journal of Logistics Management, 130-154.

Flynn, B. B., Huo, B., \& Zhao, X. (2010). The impact of supply chain integration on performance: A contingency and configuration approach. Journal of Operations Management, 58-71.

Huo, B., Ye, Y. Z., \& Zhu, K. (2016). Supply Chain Quality Integration: A txonomy perspective. Int. J. Production Economics, 111.

Instituto de Investigaciones Económicas y Sociales. UNAH. (n.d.). Producción de Alto Rendimiento (PAR). Retrieved Julio 2017, from http://www.iies-unah.org/par/

Jean, R.-J. “. (2007). The ambiguous relationship of ICT and organizational performance: a literature review. critical perspectives on international business, 306321.

Leuschner, R., Rogers, D. S., \& Charvet, F. F. (2013). A Meta - Analysis of supply chain integration and firm performance. Journal of Supply Chain Management, 35-57.

Machuca, J. A., Ortega Jiménez, C. H., Pedro, G.-V., \& Pérez Diéz de los Rios, J. L. (2011). Do technology and manufacturing strategy links enhance operational performance? Empirical research in the auto supplier sector. Int. J. Production Economics, 541-550.

Narayanan, S., Jayaraman, V., Luo, Y., \& Swaminathan, J. M. (2011). The antecedents of process integration in business process outsourcing and its effect on firm performance. Journal of Operations Management, 3-16.

Ortega Jimenez, C. H. (2016). Memoria Científico - Técnica del Proyecto . COMPETITIVIDAD Y PRODUCTIVIDAD: PRODUCCIÓN (BIENES Y SERVICIO) DE ALTO RENDIMIENTO (PAR). Tegucigalpa, Honduras. 


\section{INTEGRACIÓN Y TIC EN HOTELES: IMPORTANCIA DE SU IMPLEMENTACIÓN CONJUNTA}

Revista Economía y Administración, Vol. 9, No. 1, 2018

Dalmatia: An Analysis From Guest

Raudales García, N. R. (2013). Acceso a las tecnologías de información y la reducción de inequidades. Revista Economía y Empresa (E\&A), 147-160.

Rodriguez-Rodriguez, R., \& Leon, R. D.

(2016). Social network analysis and

supply chain management. International

Journal of producton management and

engineering, 35-40.

Rosenzweig, E. D., Roth, A. V., \& Dean Jr., J. W. (2003). The influence of an integration strategy on competitive capabilities and business performance: An exploratory study of consumer products manufacturers. Journal of Operations Management, 437-456.

Saunders, M. N., Altinay, L., \& Riordan, K. (2009). La gestión de la integración cultural posterior a la fusión: implicaciones de la industria hotelera. The Service Industry Journal, 1359-1375.

Šerić, M., \& Gil-Saura, I. (2012). ICT, IMC, and Brand Equity in High-Quality Hotels of
Perceptions. Journal of Hospitality Marketing \& Management, 821-851.

Šeric, M., Gil-Saura, I., \& Alejandro, M.-D. (2016). Can advanced technology affect customer-based brand equity in service firms? An empirical study in upscale hotels. Journal of Service Theory and Practice.

Sigala, M. (2005). Integrating customer relationship management in hotel operations: managerial and operational implications. Hospitality management, 391-413.

Sirirak, S., Islam, N., \& Ba, K. D. (2011). Does ICT adoption enhance hotel performance? Journal of Hospitality and Tourism Technology, 34-49.

Soltani, Z., \& Navimipour, N. J. (2016). Customer relationship management mechanisms: A systematic review of the state of the art literature and recommendations for future research. Computers in Human Behavior, 667-688.

Stank, T. P., Daugherty, P. J., \& Ellinger, A. E. (1999). Marketing/Logistics 


\section{INTEGRACIÓN Y TIC EN HOTELES: IMPORTANCIA DE SU IMPLEMENTACIÓN CONJUNTA}

Revista Economía y Administración, Vol. 9, No. 1, 2018

integration and firm performance. The

International Journal of Logistics

Management, Vol 10 p 11-24.

Wong, C. Y., Boon-Itt, S., \& Wong, C. W.

(2011). The contingency effects of

environmental uncertainty on the

relationship between supply chain

integration and operational performance.

Journal of Operations Management, 604

615.

Xiaoqiu Ma, J., Buhalis, D., \& Song, H.

(2003). ICTs and Internet adoption in

China's tourism industry. International

Journal of Information Management,

451-467. 